\section{MS12-P7 Isolation, purification and crystallization of a novel bidirectional NK activating ligand CD160 in complex with herpesvirus entry mediator HVEM \\ Ivana Nemčovičová ${ }^{1,2}$, Marek Nemčovič ${ }^{3}$, Marcela Kúdelová ${ }^{1}$, Dirk M. Zajonc ${ }^{2}$}

1. Biomedical Research Center at the Slovak Academy of Sciences, Dúbravská cesta 9, Bratislava, SK 84505, Slovakia

2. La Jolla Institute, 9420 Athena Circle, La Jolla, CA 92037, United States

3. Institute of Chemistry at the Slovak Academy of Sciences, Dúbravská cesta 9, Bratislava, SK 84505, Slovakia

email: ivana.nemcovicova@savba.sk

Many pathogens have evolved countermeasures to avoid detection and clearance by natural killer (NK) cells $[\mathbf{1 , 2}]$, however, regulatory mechanisms limiting cytokine activation of NK cells that reduce non-specific tissue damage remain poorly defined. CD160 is a $27 \mathrm{kDa}$ glycoprotein which was initially identified with the monoclonal antibody BY55 [2]. Its expression is tightly associated with peripheral blood NK cells and CD8 T lymphocytes with cytolytic effector activity $[\mathbf{2 , 3}, \mathbf{4}]$. The cDNA sequence of CD160 predicts a cysteine-rich, glycosylphosphatidylinositol-(GPI)-anchored protein of 181 amino acids with a single Ig-like domain weakly homologous to KIR2DL4 molecule [Fig.1C]. It was found that TNF receptor herpesvirus entry mediator (HVEM) preferentially engages CD160 trimer to costimulate activation, while a viral ortholog of HVEM specifically binds to $\mathrm{B}$ and $\mathrm{T}$ lymphocyte attenuator (BTLA) to suppress this signaling. Thus, regulation of CD160 bidirectional binding may represent a common mechanism of immune regulation targeted by multiple pathogens, which by extension is a potential target for therapeutic manipulation. We have found that CD160 is expressed at the cell surface as a tightly disulfide-linked multimer. The homology model of CD160 antigen domain [Fig.1B] shows cysteine-rich region that was found to be responsible for CD160 tight-timer formation even under reduced conditions. CD160 trimer forms stable complex with HVEM, while monomeric form refused to binds its cognate ligand. We have attempted crystallization of HVEM and CD160 molecules in singles, as well as in complex. Protein crystals shown in Fig.1A were grown by using PEG ion and Morpheus crystal screens and successfully tested for X-ray diffraction.

IN is Marie Curie Fellow financed by Programme SASPRO, co-funded by European Union and the Slovak Academy of Sciences. The authors gratefully acknowledge the contribution of the Slovak Research and Development Agency under the project APVV-14-0839 and the contribution of the Scientific Grant Agency of the Slovak Republic under the grant 2/0103/15.

1. Vivier E, et al., Science 2011, 331, $44-49$

2. Anumanthan A, J Immunol 1998, 161(6), 2780-90

3. Agrawal S, et al., J Immunol 1999, 162(3), 1223-26

4. Klopocki E, Am. J. Hum. Gen. 2007, 80(2), 232-40

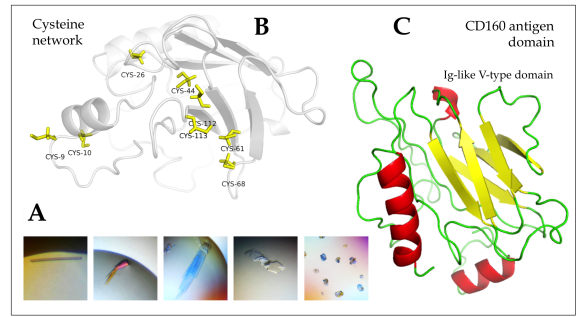

Figure 1. A, protein crystals of CD160, CD160-HVEM complex and HVEM; B, The network of intramolecular cysteines within CD160 ectodomain; C, The homology model of CD160 structure showing Ig-like V-set type domain and surrounding helice.

Keywords: NK cells, CD160 antigen, herpesvirus entry mediator HVEM 